\title{
Supporting The DevelopMent of Problem Solving SkILlS IN AN INTEGRATED ELECTROMAGNETICS AND VECTOR Calculus Course
}

\author{
Carol P. Jaeger, Philip D. Loewen, and Negar M. Harandi \\ The University of British Columbia \\ carolj@apsc.ubc.ca
}

\begin{abstract}
Electromagnetics and vector calculus are taught as an integrated course in the Electrical Engineering program at UBC. In this paper, the course structure is described, and unique features are highlighted. A key goal of the course is to help students develop problem solving skills. To assist students in building these skills, a blended classroom approach has been adopted to allow an increase in discussion and problem solving activities in the classroom. Results of student assessment and a summary of student feedback on the teaching technologies and activities incorporated into the course are reported.
\end{abstract}

Keywords: electromagnetics, vector calculus, blended learning, problem solving, teaching technologies, WeBWorK, videos, in-class polling

\section{INTRODUCTION}

At the University of British Columbia (UBC), the Department of Electrical and Computer Engineering has partnered with the Department of Mathematics to create and deliver a course that truly integrates the teaching and application of vector calculus to the study of electromagnetics. The course is delivered in a blended format and incorporates a variety of technology-based tools to motivate student engagement and enhance student learning. Using a blended classroom approach has made it possible to give additional time in class for students to work through problems collaboratively and for the instructors to work individually with students in class. In this paper the motivation behind the development of the course and a report on student response and efficacy of various tools employed in the course is presented.

\section{COURSE DETAILS}

\subsection{History}

Prior to the introduction of the new course, students in Electrical Engineering took a combined multivariable and vector calculus course (MATH 263, 4 credits), followed by an engineering electromagnetics course (EECE 261, 3 credits). There was no formal connection between the instructional teams of the two courses. The majority of other engineering programs contain a traditional multivariable calculus course (MATH 253, 3 credits) with no formal coverage of vector calculus. Students reported minimal retention of the vector calculus components of MATH 263, and instructors of EECE 261 consequently dedicated significant lecture time to review of vector calculus concepts.

In 2009 the Electrical and Computer Engineering department struck a working group to review the content of both the math and electromagnetics course. The group proposed that the coverage of vector calculus could be strengthened by integrating it with the electromagnetics course. Further, it was determined that by regrouping the topics, a net reduction of 1 credit could be achieved. Effective since the 2011 academic year, students now take MATH 253 alongside students in other programs, followed by an integrated combination of vector calculus (MATH 264, 1 credit) and engineering electromagnetics (ELEC 211, 2 credits). MATH 264 and ELEC 211 retain separate codes for administrative purposes, but are taught as a single 3 -credit course. Students register for both courses. Therefore, for the balance of this paper, the two courses will be referred to as 'the course'. At the time of the curriculum change, we were not aware of any similar formal integrations of these two topics at other institutions.

\subsection{Learning Goals}

A key learning goal for the course is for students to be able to develop problem-analysis and problem solving skills (CEAB graduate attribute \#2) [1]. Specifically, in this course the goal is for students to develop their ability to create idealized models of different devices or structures common in electrical engineering, construct appropriate mathematical models for the system in question, and then evaluate the equations that were developed to arrive at a solution to the stated problem. As an example, second year students often struggle to make the transition from solving 
a given integral to building the relevant integral to solve a problem. The integrated course is designed to give students additional support in developing their skills in model selection and application.

At the content level, the course learning goals are focused on the understanding and application of vector calculus and electrostatic and magnetostatic laws. The course syllabus states that student should be able to:

- Perform mathematical operations with vector quantities

- Solve line, surface, and volume integrals in multiple coordinate systems

- Apply Divergence and Stokes' theorems correctly in problem solving

- Solve for the force on charged structures in the presence of electric fields

- Solve for the electric field at a point due to a variety of charge distributions

- Apply Gauss' law in the solution of electric field distributions resulting from charge distributions

- Apply Ampere's law to derive magnetic field distributions resulting from current distributions

- Calculate the displacement current in simple circuits

- Analyze the behavior of simple conducting structures in the presence of a time-varying magnetic field

- Use boundary conditions to determine the effect of different materials on electric and magnetic fields

- Evaluate the capacitance or inductance of various structures

- Explain the principal of operation of a variety of electromagnetic devices

- Understand and apply Maxwell's equations

\subsection{Course Structure}

The course is structured as a typical 3 lecture hour per week offering, delivered in two 90-minute sessions, with an additional 2-hour tutorial on alternating weeks. The course has an annual enrolment of approximately 190 students, divided into two lecture sections and sharing a single tutorial section. The tutorial section is primarily used to administer a series of four tests evenly spaced throughout the term.

Two instructors are assigned to the course annually, one from the Department of Mathematics, and one from the Department of Electrical and Computer Engineering. The lecture plan is determined collaboratively between the two instructors, and students are informed that either instructor may deliver any particular lecture. Where appropriate, both instructors co-present material. .

One of the primary goals for the course is to give students formal instruction on vector calculus concepts in the context of their application to electromagnetics problems. As such, the math content is more heavily weighted towards the beginning of the course, with the number of math-focused lectures tailing off as the term progresses. The lecture plan is developed by first planning the sequence of electromagnetics topics to be introduced and interleaving the relevant vector calculus concepts so that they can immediately be applied to typical electro- and magnetostatic structures.

\subsection{Teaching Technologies}

Several teaching technologies have been integrated into the course in the past three years. These include: online homework submission, a discussion forum, custom video content, and in class polling software. Each of these technologies are described in turn.

2.4.1. WeBWorK. WeBWorK is an online homework submission platform developed by the Mathematical Association of America [2]. WeBWorK allows for randomized variables within problem statements so that each student receives a unique version of each question. WeBWorK was first introduced in the course three years ago. It has allowed homework to become an official part of the assessment strategy, worth $10 \%$ of the term grade. Students get unlimited attempts at problems, and are encouraged to work together. Because final answers cannot be copied students must discuss their problem solving techniques if they are to help each other. WeBWorK is an open source platform and sharing of coded problems is encouraged. An open problem library of some 35,000 problems exists, however, very few entries in the existing library are dedicated to engineering problems. Despite this fact, several instructors of engineering courses at UBC have found value in this tool and have adopted it in their courses [e.g. 3]. Additionally, a team of instructors has received funding from the University to develop and code a new library of engineering-specific questions across a range of disciplines over the coming year.

2.4.2. Piazza. Piazza is an online discussion forum used in a variety of courses at UBC and elsewhere. It has been made available to students in this course for two years now. The Piazza forum is monitored informally by one instructor but is primarily made available to students to ask and answer questions peer-to-peer. No formal expectations have been set around the use of Piazza by students or for the frequency with which an instructor might review and respond to posts.

2.4.3. ViDeX. A new video viewing application was trialed for the 2018 offering of the course. ViDeX [4], developed at UBC, was adopted as the viewing platform for a set of pre-week videos. ViDeX allows students to bookmark content, highlight favorite passages in videos and make notes. It allows instructors to collect data on viewing patterns, with more sophisticated analytical tools still under development. 
Screencast style videos have been used on an ad hoc basis in the course in previous years to address common student questions or to provide additional details on subjects of interest to students when class time did not permit sufficient discussion. This year a concerted effort was made to develop and release one video every week for the duration of the course. Each video covered theoretical material central to the topics for the coming week. This was a fundamental shift in the purpose of video content employed in the course.

A weekly flow for the course was structured to be very similar to that of APSC $100 / 101$ [5], which $75 \%$ of the class has previously taken, and which also used weekly video content ahead of each week. Consistent naming of the pre-week preparation ('Getting Ready For The Week'), was used to highlight the similarities in structure and expectations between APSC 100/101 and this course. Short quizzes were used to encourage students to view the weekly videos. The deadline for each 'mini-quiz', which consisted of two or three short questions requiring direct application of the material in the video, was $8 \mathrm{am}$ on the day of the first lecture of the week. By moving a subset of the introductory material each week to video presentations, the difficulty level of practice problems introduced in class was increased.

2.4.4. Mentimeter. Also new for this year was the incorporation of a web based polling application called Mentimeter [6] into classes to encourage participation. The poll questions are easy to construct, and are easily viewed on a smartphone, tablet, or laptop. Students were not required to create an account or identify themselves in order to use Mentimeter. While regular attendance was encouraged, Mentimeter was used in an anonymous polling format only. Questions were used to stimulate discussion and help resolve conceptual errors. Students seemed comfortable using the software and having a way to submit answers anonymously seemed to promote student discussion.

\section{RESULTS}

\subsection{Diagnostic Test}

A diagnostic test, compromised of 10 electromagnetics (E\&M) and 5 vector calculus (VC) multiple choice (MC) questions, was constructed based on learning objectives of the course's pre-requisites, as well as published literature [7]. The test was administered on the first lecture of the term. The students had not been informed about the diagnostic quiz, but were assured that the test was not for credit. A total of 187 students took the test in two sessions. The average score in the class was $40 \%$ for the E\&M, and $58 \%$ for the VC questions. Student performance on the qualitative $\mathrm{E} \& \mathrm{M}$ questions improved over the course of the semester, when measured against a different set of $10 \mathrm{MC}$ qualitative questions on the final exam: the class averaged $59 \%$.

Four of the questions on the diagnostic test were directly adapted from Singh [7], identified as Q3, Q4, Q5 and Q7 in that paper. Table 1 compares the performance of our students with those of first-year introductory calculus-based physics courses described in [7]. The two columns "Pre" and "Post" indicate quiz scores before and after formal instruction. The results were reasonable given that they had completed the equivalent first-year course at least 8 months prior to completing this diagnostic quiz.

Table 1. Students' average performance on four E\&M diagnostic questions adapted from Singh [7]. Results in our class are compared to the pre- and post-instruction data reported by Singh.

\begin{tabular}{|c|c|c|c|}
\cline { 2 - 4 } \multicolumn{1}{c|}{} & ELEC 211 & "Pre" $[z]$ & "Post" $[z]$ \\
\hline Q3 & $59 \%$ & $39 \%$ & $39 \%$ \\
\hline Q4 & $47 \%$ & $17 \%$ & $64 \%$ \\
\hline Q5 & $58 \%$ & $42 \%$ & $47 \%$ \\
\hline Q7 & $37 \%$ & $16 \%$ & $49 \%$ \\
\hline
\end{tabular}

\subsection{Student Survey}

A short survey was administered to the students at the midpoint of the course to collect feedback on various aspects of the course structure and teaching tools. There were 57 responses submitted from a class size of 194 . Student response to the perceived usefulness of each teaching technology or activity is summarized in Fig. 1. Overall, students responded positively to most technologies and activities in the course, with Piazza, WeBWorK, and in-class problem solving getting the highest overall ratings as useful components in the course.

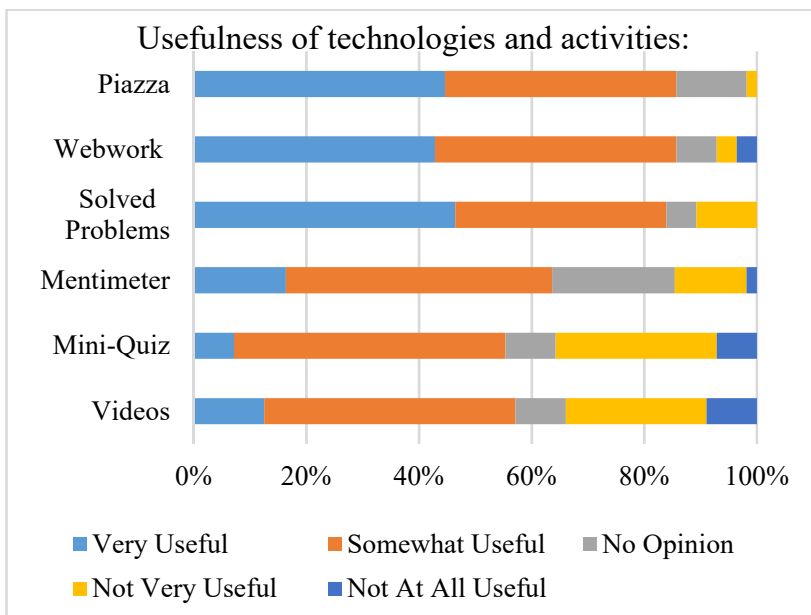

Fig. 1. Student feedback on usefulness of technologies and activities used in the 2018 class as indicated in the the midsemester survey. 


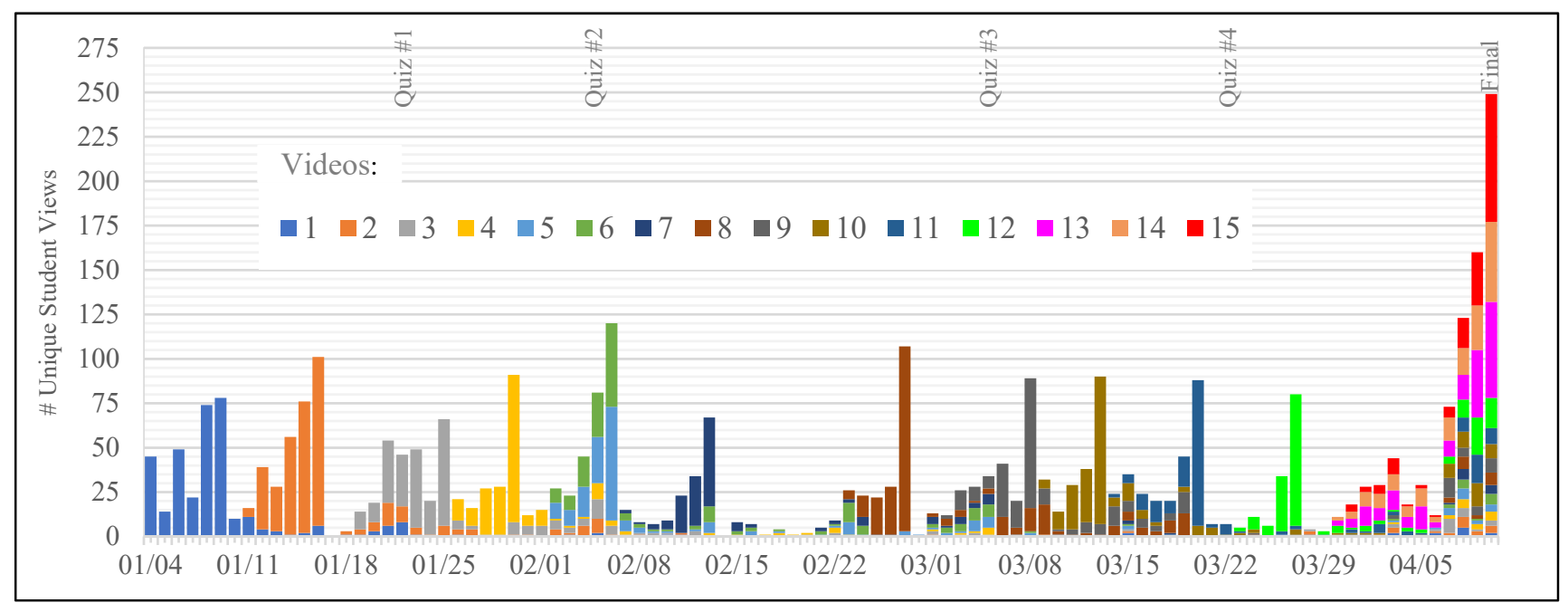

Fig. 2. Unique student views over time recorded on the ViDeX platform. Fifteen videos are encoded in different colors. Each date on the x-axis shows a release date for one or two weekly videos which were due two days before the next release. Some occasional adjustment to the due date was made (on $01 / 25$ and $03 / 08$ ) to adapt to midterm exam schedules.

In response to "If you could increase/decrease the time spent in class on any one thing, what would it be?" students generally indicated that they would prefer less lecture time, and instead would prefer to see more problems solved by the instructor during class time. Student attitude towards solving problems with their peers in class was divided, and although $65 \%$ of respondents were satisfied with having the Mentimeter questions, about one in five students indicated that they would prefer to have less time devoted to them. See Table 2. We suspect these students had difficulty establishing a direct link between the qualitative discussions in the class, and the exam assessments, specifically at the mid-point of the semester, and so were favoring solved problems over such class activities. A more direct question in the survey which targeted student attitudes towards the Mentimeter questions is discussed later in section 3.5.

Table 2. Student feedback on their preference for the amount of time spent on different classroom activities.

\begin{tabular}{|l|l|l|l|l|}
\cline { 2 - 5 } \multicolumn{1}{c|}{} & $\begin{array}{l}\text { Menti- } \\
\text { meter }\end{array}$ & $\begin{array}{l}\text { Lecture } \\
\text { time }\end{array}$ & $\begin{array}{l}\text { Instructor } \\
\text { Problems }\end{array}$ & $\begin{array}{l}\text { Student } \\
\text { Problems }\end{array}$ \\
\hline Increase & +1.9 & +11.5 & +59.6 & +26.9 \\
\hline Decrease & -20.0 & -50.0 & -6.0 & -24.0 \\
\hline Overall & -18.1 & -38.5 & +53.6 & +2.9 \\
\hline
\end{tabular}

\subsection{WeBWorK}

Survey data showed $85 \%$ of students found WeBWorK questions to be either very useful or somewhat useful, and $89 \%$ of students who responded felt that the number of questions assigned each week was about right. Interestingly, while a number of students commented in open text fields that they would like more practice problems, only $31 \%$ responded that they would do extra problems "most or all of the time" if the problems were not for credit.

Data from the 2016, 2017 and 2018 course offerings showed that, averaged across 11 problem sets, the majority of students were diligent about doing homework problems. Table 3 shows the percentage of students who persisted until they scored, on average, $80 \%$ and $100 \%$ across eleven homework sets during the term. Additionally, there was a modest but positive correlation between homework scores and final grades. $\mathrm{R}^{2}$ values for each offering are also summarized in Table 3 .

Table 3. Students' performance in WeBWorK assignments.

\begin{tabular}{|c|c|c|c|}
\hline Year & $\begin{array}{c}\text { \% students } \\
\text { scoring } \mathbf{1 0 0} \%\end{array}$ & $\begin{array}{c}\text { \% students } \\
\text { scoring } \mathbf{8 0 \%}\end{array}$ & $\begin{array}{c}\text { Homework v. } \\
\text { final grade } \\
\mathbf{R}^{\mathbf{2}} \text { value }\end{array}$ \\
\hline $\mathbf{2 0 1 6}$ & $28 \%$ & $70 \%$ & 0.47 \\
\hline $\mathbf{2 0 1 7}$ & $65 \%$ & $80 \%$ & 0.25 \\
\hline $\mathbf{2 0 1 8}$ & $45 \%$ & $78 \%$ & 0.46 \\
\hline
\end{tabular}

\subsection{ViDeX}

Figure 2 shows the use of pre-class videos recorded on the ViDeX platform. The time line in the figure starts on the first day of the 2018 class and ends on the day of the final exam. Fifteen videos, encoded in different colors, were administered in total, with at least one video each week. Videos were released every Thursday and the miniquiz related to each video closed on the following Tuesday. No video was assigned on the week of the reading break (February 18-22). Most videos from the second half of the 
course were revisited before the final exam. Overall, videos had a range of 91 (last video) to 162 (first video) unique student views during the semester.

\subsection{Mentimeter}

We asked students what they thought about the Mentimeter questions. This survey question allowed multiple selections, and 53 respondents entered a total of 141 selections. The number of responses per choice is shown in Table 4. This data shows that a majority of the students who completed the survey appeared to find value in having Mentimeter in the classroom.

Table 4. Students' feedback on use of the Mentimeter questions as indicated in the 2018 mid-semester survey.

\begin{tabular}{|l|c|}
\hline $\begin{array}{l}\text { Mentimeter question feedback } \\
\text { (select all that apply) }\end{array}$ & $\begin{array}{c}\text { \# of } \\
\text { responses }\end{array}$ \\
\hline I find they make the class more engaging & 36 \\
\hline I like that they are anonymous and not for credit & 32 \\
\hline I like them, they help me understand the concepts & 28 \\
\hline I like working with classmates to answer them & 22 \\
\hline I don't mind them but I'm not excited by them & 16 \\
\hline I wish they were for credit & 1 \\
\hline I don't like the Mentimeter activities & 1 \\
\hline Other (please explain) & 5 \\
\hline
\end{tabular}

\subsection{Concept Inventory}

For the last 3 years, we have included an identical set of $10 \mathrm{MC}$ questions selected from [8] and used by permission. No deductions are made for incorrect or unanswered questions. The class average on these 10 questions has remained steady at $60 \pm 1 \%$ with a standard deviation of $21 \%$ over the 3 year span, however, the correlation between scores on the $\mathrm{MC}$ questions and the rest of the exam has improved modestly, with $\mathrm{R}^{2}$ values of $0.16,0.17$, and 0.25 . Final grades in the course have also been relatively steady, but are also more difficult to compare given that, other than the $10 \mathrm{MC}$ questions described here, all other quiz and final exam questions were different every year, and removing variation due to question difficulty level and variations in grading styles across the instructional team would be difficult to achieve.

\section{DISCUSSION}

A variety of interesting results have emerged from the data collected during the term. Perhaps the most significant result is that, despite additional classroom time being dedicated to understanding the 'big-picture' concepts through use of qualitative concept questions, longitudinal data collected across 3 years in which a consistent set of 10 $\mathrm{MC}$ questions were included on the final examination showed no change in the class average on the set of questions.

From both the student and instructor perspective, addition of WeBWorK-based homework for course credit has been very successful. The majority of students make an effort to attempt if not complete the weekly homework questions, and students routinely discuss aspects of the problems with the instructional team and with each other. At UBC a concerted effort to increase the inventory of WeBWorK questions and reduce the reliance on either textbooks or fee-based homework submission software is underway.

The addition of video based content appears to be mixed. While students clearly indicate that they would prefer to see more class time devoted to examples and solved problems, data on the number of unique viewers indicates that not all students are using the videos as a resource. It is worthwhile noting that, while some summary notes were provided, in order to encourage students to prepare for the week in time to make best use of the class time, video content was only reviewed in class in a very brief way to set context for the classroom activities. It is possible that students were relying on the recommended textbook for the course, as students were provided with references to appropriate sections of the text with each video release. In general the adoption of blended learning and flipped classroom teaching techniques should be adaptable to a variety of student learning styles. Further, it should support the development of skills needed to identify gaps in ones learnings and to engage in independent learning. Thus, in this course we continue to work to foster lifelong learning as well as problem solving skills in our students. One potential downside of moving traditional lecture content into video format is that it did seem to have an effect on attendance. However, even though attendance was lower than is typical in one of two sections, the level of engagement of the students who attended was high.

The data from table 2 summarizing student responses to what they would like to see more of or less of in class is perhaps not surprising, but still very interesting. Overall, it suggests that students value seeing as many worked examples as possible, preferring this over discussion of theory. However, students also indicate that given a choice, they would prefer to watch an instructor-led presentation of example problems rather than work on problems themselves or in groups. While easier to watch rather than do, students continue to struggle to solve new problems in an exam setting. In the current course structure, theory is still presented in video format and available in texts and notes, and concepts are explored in class, but students would likely benefit even further by 
taking full advantage of problem solving exercises during class time.

The Mentimeter experiment was a success from the instructor's perspective, bringing considerable enthusiasm and engagement to the classroom. We found that 3 or 4 short questions that led students through a sequence of related ideas was optimal in a 90 minute lecture. Anecdotally, most students in the class were on task with these questions, and tended to work in small groups to debate the answers to each question. We will continue use of this tool in future years.

\section{CONCLUSIONS AND FUTURE WORK}

A strategy for supporting the development of problem solving skills in a combined electromagnetics and vector calculus course was presented. A variety of technologybased tools and activities were employed in the course, each of which was reviewed in this paper. While measurable results in the form of increased course grades remain elusive, early signs are that these tools and strategies could be developed further to guide students. Each of the technologies and activities incorporated into the course were sufficiently well received by both students and instructors to warrant their ongoing use in the course.

Students had access to a variety of resource materials in this course, which gives more flexibility in learning styles, but makes it harder to track which approaches or activities may have the most concrete results for students. In future offerings of this course, we hope to find ways to better track the usage of each of the new activities and their impact on student achievement in the course. Student preference for passive rather than active participation in problem solving and examples of application of theory is unsurprising, but likely a key factor in making significant improvements in student problem solving skills. This is one aspect that the authors intend to explore actively prior to the next course offering.

\section{Acknowledgements}

We would like to acknowledge the UBC Department of Electrical and Computer Engineering and the UBC Teaching and Learning Enhancement Fund for supporting course development and renewal through the funding of $\mathrm{N}$. Harandi as a Teaching and Learning Fellow.

\section{References}

[1] Canadian Engineering Accreditation Board, Engineers Canada, Accreditation Criteria and Procedures, revised October 2017.

[2] Mathematical Association of America: Webwork homepage. http://webwork.maa.org/

[3] A.G. D'Entrement, P.J. Walls, and P.A. Cripton. Student Feedback and Problem Development for WeBWorK in a Second-year Mechanical Engineering Program, in Proc. Of CEEA 2017, 6 pp., Toronto, 2017.

[4] Fong, M., Dodson, S., Zhang, X., Roll, I., \& Fels S. (in press). ViDeX: A platform for personalizing educational videos. In Proceedings of the 2018 ACM/IEEE Joint Conference on Digital Libraries (JCDL).

[5] P.M. Ostafichuk, C.P. Jaeger, J. Nakane, S. Nesbit, N. Ellis, J. Sibley, "Redesigning the UBC First Year Introduction to Engineering: Successes and Challenges," in Proc. of CEEA 2016, 8 pp., Halifax, 2016.

[6] Mentimeter. Available online at www.mentimeter.com. [7] Singh, C., 2017. Improving Student Understanding of Magnetism. arXiv preprint arXiv:1701.01523.

[8] B. Notaros.

http://www.foundationcoalition.org/home/keycomponents /concept/electromagnetics.html 